\title{
SUPPROTING INFORMATION
}

\section{Next-generation antimicrobial-resistance surveillance system based on internet-of-things and microfluidic technique}

\author{
Luyao $\mathrm{Ma}^{1,2}$, Weidong $\mathrm{He}^{3}$, Marlen Petersen ${ }^{1}$, Keng C. Chou ${ }^{4}$, Xiaonan $\mathrm{Lu}^{1,2, *}$
}

${ }^{1}$ Food, Nutrition and Health Program, Faculty of Land and Food Systems, The University of British Columbia, Vancouver, BC, V6T 1Z4, Canada

${ }^{2}$ Department of Food Science and Agricultural Chemistry, Faculty of Agricultural and Environmental Sciences, McGill University, Sainte-Anne-de-Bellevue, QC, H9X 3V9, Canada

${ }^{3}$ College of Computer Science, Chongqing University, Chongqing, 400044, China

${ }^{4}$ Department of Chemistry, Faculty of Science, The University of British Columbia, Vancouver, V6T 1Z1, Canada

* Corresponding author (X. L.): email: xiaonan.lu@mcgill.ca; phone: +1 514-398-7921 


\section{EXPERIMENTAL SECTION}

\section{Antibiotics, bacterial strains, and growth conditions}

Tetracycline hydrochloride, ciprofloxacin, and erythromycin (Sigma-Aldrich, Canada) were used as the representative antibiotics in this study. Antibiotic stock solutions were prepared by dissolving the antibiotic powder into appropriate solvents to achieve a concentration of $1,280 \mathrm{mg} / \mathrm{mL}$, followed by filtrating through sterile nylon syringe filters $(0.2-\mu \mathrm{m}$ pore size; VWR, Canada). Specifically, $70 \%$ ethanol aqueous solution, $0.1 \mathrm{M} \mathrm{HCl}$ aqueous solution, and ethanol were used as solvents for tetracycline hydrochloride, ciprofloxacin, and erythromycin, respectively. The stock solutions were stored in dark at $-20^{\circ} \mathrm{C}$ for up to 1 month. Before antimicrobial susceptibility testing, antibiotic working solutions were freshly prepared by diluting stock solutions in sterile $\mathrm{ddH}_{2} \mathrm{O}$ to reach the defined concentrations. The antibiotic working solutions were then deposited onto a sterile paper disc (in-house punched, $\varnothing=1.5 \mathrm{~mm}$, Whatman qualitative filter paper, Grade 1; Sigma- Aldrich, Canada).

Three Campylobacter strains were selected as the reference strains to assess the performance of the on-chip antimicrobial susceptibility testing, including $C$. jejuni $\mathrm{F} 38011$ (human clinical isolate), $C$. jejuni ATCC 33560 (bovine feces isolate), and C. coli 314 (pig isolate). Bacterial strains were routinely cultivated on Mueller Hinton (MH) agar (BD Difco; Fisher Scientific, Canada) supplemented with 5\% (v/v) defibrinated sheep blood (Abbott, Canada). The agar plates were incubated in the microaerobic condition $\left(85 \% \mathrm{~N}_{2}, 10 \% \mathrm{CO}_{2}, 5 \% \mathrm{O}_{2}\right)$ at $37^{\circ} \mathrm{C}$ for 2 days before subculturing to fresh $\mathrm{MH}$ blood agar plates. To prepare bacterial overnight culture, a few bacterial colonies were transferred from $\mathrm{MH}$ blood agar plates to fresh MH broth (BD Difco; Fisher Scientific, Canada) and shaken at $175 \mathrm{rpm}$ and $37^{\circ} \mathrm{C}$ in the microaerobic condition for 16-18 $\mathrm{h}$.

\section{Fabrication of microfluidic chips}

Microfluidic chips were designed in AutoCAD (Version 2020; Autodesk, USA); they comprised one plain glass layer for sealing and observation, one polydimethylsiloxane (PDMS) middle layer for bacterial incubation, and one PDMS bottom layer for sealing and sample injection. Fig. S4 shows the patterns of the middle and bottom layers, which were printed onto a photomask by CAD/Art Services (Bandon, OR, USA) for silicon mold microfabrication. Standard photolithographic technologies ${ }^{1}$ were performed to fabricate the device features on a silicon wafer ( $\varnothing=100 \mathrm{~mm}$; University Wafer, USA) on which an 80- $\mu$ m-thick SU-8 2035 photoresist (Microchem, USA) was applied. Once the SU-8 based 
silicon mold was obtained, soft lithography was applied to produce microfluidic chips ${ }^{2}$. PDMS monomer and curing agent (Dow Sylgard 184 silicone encapsulant clear kit; Ellsworth Adhesives, USA) at a weight ratio of 10:1 were mixed and degassed in a vacuum desiccator at the ambient temperature $\left(22^{\circ} \mathrm{C}\right)$. Twenty-two grams of PDMS mixture was poured onto the silicon molds and cured for $35 \mathrm{~min}$ on a hot plate at $80^{\circ} \mathrm{C}$. The cured PDMS slabs were peeled off from the silicon molds, and Miltex ${ }^{\circledR}$ biopsy punches (Ted Pella Inc., USA) were used to create bacterial incubation chambers (middle layer), inlets/outlets (middle and bottom layers), and air vents (bottom layer) on PDMS slabs. Subsequently, the PDMS middle and bottom layers were treated with oxygen plasma (plasma cleaner PDC32G; Harrick Plasma, USA) for $5 \mathrm{~min}$ and bound together. For bacterial studies, a piece of PVDF membrane ( $\varnothing=4$ mm, Amersham Hybond P 0.45; VWR, Canada) was deposited into each bacterial incubation chamber, followed by adding an antibiotic-preloaded paper disc and $20-\mu \mathrm{L}$ chromogenic agar medium (CHROMagar Campylobacter; CHROMagar, France). Lastly, a Corning ${ }^{\mathrm{TM}}$ plain glass microscope slide (Fisher Scientific, Canada) was assembled to the PDMS middle/bottom hybrid slab using the oxygen plasma-assisted method.

\section{IoT-based colorimetric sensing system for bacterial on-chip test}

To continuously monitor the growth of Campylobacter in the microfluidic chips, a colorimetric sensing system was constructed as shown in Fig. 2. This home-built system consists of three compartments, including a portable incubator, a camera, and a WiFi-capable laptop (Fig. 2A). The incubator was fabricated using a sealed plastic box with dimensions of $20 \times 12 \times 7.5 \mathrm{~cm}^{3}$ and a weight of $323 \mathrm{~g}$ (Fig. 2B). Since Campylobacter has fastidious growth requirements on temperature and atmosphere ${ }^{3}$, the incubator was constructed to achieve an optimal growth temperature of $42^{\circ} \mathrm{C}$ and a $\mathrm{CO}_{2}$ concentration of $\sim 10 \%$. An aluminum plate was used as a heat reservoir for incubation. Four pieces of polyimide foil adhesive heaters (HT10K; Thorlabs, USA) were attached to the bottom of the aluminum plate and connected to a heater controller (TC200; Thorlabs, USA). A temperature probe (TSP01; Thorlabs, USA) was attached to the center of the aluminum plate to measure the real-time temperature for a feedback loop, maintaining the temperature of the aluminum plate at $42 \pm 0.5^{\circ} \mathrm{C}$. The concentration of $\mathrm{CO}_{2}$ (industrial pure gas; Praxair, Canada) was controlled by a 2-way normally closed electric gas valve. The open/close of the gas valve was determined by $\mathrm{CO}_{2}$ concentration monitored by a $\mathrm{CO}_{2}$ sensor (GC-0006; CO2Meter, USA). A micro gas pump (PMP-0010; CO2Meter, USA) was installed inside the incubator to circulate $\mathrm{CO}_{2}$ into the incubator. The aluminum plate has four pockets ( 5 
$\mathrm{cm} \times 8 \mathrm{~cm}$ ) with a depth of $2 \mathrm{~mm}$ to house the microfluidic chips. These pockets were filled with sterile water to maintain sufficient humidity in the incubator during bacterial cultivation. The relative humidity was recorded by a humidity sensor (TSP01; Thorlabs, USA). To monitor the colorimetric reaction between Campylobacter and chromogenic agar medium, a camera (A6981310; Microsoft, USA) was installed at the fixed distance $(9 \mathrm{~cm})$ and angle $\left(90^{\circ}\right)$ from the incubator to obtain repeatable results under the same conditions. An LED array was used to provide stable and uniform illumination. All electronics were controlled by a self-developed LabVIEW program (National Instruments, USA). Once the images were obtained, machine learning algorithms were applied for image analysis. The on-chip results were transmitted from the local laptop to Tencent Cloud server (Tencent, China) via WiFi.

\section{Preparation of training data set}

On-chip AST is based on the colorimetric reaction between Campylobacter cells and chromogenic agar medium. If the tested Campylobacter survived from certain antibiotic classes and concentrations, chromogenic agar in the microfluidic chips had a color change from light yellow to red; otherwise, no color change could be observed. To train the machine learning algorithms on image analysis, we firstly collected a total of 6,022 images to represent negative (i.e, inhibition) and positive (i.e., growth) signals. C. jejuni F38011 overnight culture (adjusted to $10^{8} \mathrm{CFU} / \mathrm{mL}$ in $\mathrm{MH}$ broth) was prepared as a positive sample, while sterile MH broth was used as a negative sample. To ensure the color change of $C$. jejuni culture, only chromogenic agar without the presence of antibiotics was loaded into the microfluidic chip. Bacterial culture or $\mathrm{MH}$ broth was injected into microfluidic chips using a syringe pump at the flow rate of $0.05 \mathrm{~mL} / \mathrm{min}$ until the incubation chambers were filled. The microfluidic chips were then incubated in the IoT-based colorimetric sensing system. The images of microfluidic chips were captured by the camera of the IoT-based colorimetric sensing system at the time interval of $30 \mathrm{~min}$ for up to $48 \mathrm{~h}$. All data were collected from six independent experiments on different days to include possible batch variations for data training. Each microfluidic chip contained 8 incubation chambers, and images of each chamber were saved with an image size of $50 \times 50$ pixels. The color patterns of images were visually determined and cross-validated by two well-trained personnel. As a result, 3,011 positive images and 3,011 negative images were obtained for machine learning. 


\section{Machine learning algorithms for image analysis}

Python scripts were developed to carry out machine learning algorithms. We compared the performance of three commonly used machine learning algorithms on colorimetric image analysis, including threshold model, support vector machine (SVM), and convolutional neural network-residual network 50 (CNN-ResNet50). The objective of image analysis was to classify the unknown images into one of two classes, either positive (i.e., growth) or negative (i.e., inhibition). For all algorithms, the image data set $(n=6,022)$ obtained from Section 2.4 were randomly divided into two parts: the training data set ( $80 \%$ of the data) and the test data set (the remaining 20\%). An equal number of positive and negative images were obtained in each data set to achieve an even distribution of two classes. For the threshold model, the greyscale (i.e., $0.30 \times \mathrm{R}+0.59 \times \mathrm{G}+0.11 \times \mathrm{B}$ ) of each pixel (in total $50 \times 50$ pixels) on an individual training image was summed up and used as the input data ${ }^{4}$. Next, we calculated the average greyscale value from the group of negative training images and the group of positive training images, respectively. A cut-off greyscale value was then defined by averaging the mean values of negative and positive groups so as to distinguish positive signals from negative ones. Images with the greyscale higher than the threshold were regarded as the negative class, and vice versa. For SVM, we used the radial basis function kernel to construct an optimal hyperplane with the largest margin zone between negative and positive clusters ${ }^{5}$. SVM was performed using the sklearn package on Python and the flattened greyscale of the training images was used as input data (i.e., transformed from 50×50 pixels to $1 \times 2,500$ pixels). For CNN-ResNet50, we employed a 50-layer neural network structure established by He and coauthors ${ }^{6}$ and developed the Python script by implementing multiple functions from TensorFlow (Google, USA), an open-source deep-learning algorithm library. All training images were fed into the CNN-ResNet50 model with RGB channels. An iterative process of feedforward and backpropagation learning process ${ }^{7}$ was used to optimize the parameters of the CNN-ResNet50 model, with an initial learning rate of 0.001 , a mini-batch size of 16, and the number of epochs at 10.

After generating the machine learning models, 5-fold cross-validation was carried out to assess the classification performance of each model on the test data set. The accuracy, precision, recall, and F1 score were used as performance indicators, according to the equations as follows ${ }^{8}$ :

$$
\begin{aligned}
& \text { Accuracy }=\frac{T P+T N}{T P+T N+F P+F N} \\
& \text { Precision }=\frac{T P}{T P+F P} \\
& \text { Recall }=\frac{T P}{T P+F N}
\end{aligned}
$$


$F 1$ score $=2 \times \frac{\text { precision } \times \text { recall }}{\text { precision }+ \text { recall }}$

where TP, TN, FP, FN represented true positive, true negative, false positive, and false negative, respectively. For each performance indicator, the mean and standard deviation were calculated from five repeats of validation. The mean values fall into the range of 0 to 1 , with the best performance at 1 . We selected the machine learning algorithm with the best classification performance for the following onchip AST.

\section{Antimicrobial susceptibility testing (AST)}

On-chip AST was performed to determine bacterial susceptibilities against three major campylobacteriosis prescriptions, including tetracycline, ciprofloxacin, and erythromycin ${ }^{9,10}$. For each antibiotic, we selected its CLSI susceptible breakpoint and resistant breakpoint as the tested antibiotic concentrations to determine if the bacterial samples were classified as either susceptible or resistant, according to the CLSI standard protocol with modifications ${ }^{11}$. A bacterial test without the presence of any antibiotic was used as a positive control to verify if all procedures were conducted correctly. Briefly, $0.5 \mu \mathrm{L}$ of antibiotic working solution (40× final concentrations) was loaded onto a sterile paper disc and air-dried in a biosafety cabinet. The antibiotic-loaded paper disc was placed into bacterial incubation chambers of microfluidic chips, followed by adding $20 \mu \mathrm{L}$ of chromogenic agar medium to achieve the final antibiotic concentration (Table S2). Campylobacter overnight culture was adjusted to the initial inoculation concentration of $1 \times 10^{8} \mathrm{CFU} / \mathrm{mL}$ and injected into the microfluidic chips. This bacterial concentration was higher than the detection limit of the colorimeter-based microfluidic sensors $\left(\sim 10^{2}\right.$ $\mathrm{CFU} / \mathrm{mL}$ ). The on-chip AST was incubated and monitored in the IoT-based colorimetric sensing system at $42^{\circ} \mathrm{C}$ in the microaerobic condition for $24 \mathrm{~h}$. At the end of the process, the images of on-chip AST were read by the developed Python script, where the colorimetric results of different bacteria-antibiotic combinations were simultaneously determined by the pre-trained machine learning algorithm. To validate the accuracy of on-chip AST, we compared the results to the ones obtained from the conventional broth microdilution method ${ }^{11}$. Categorical agreement rates were calculated as the performance indicator, which was equivalent to the number of isolates with the same categorical interpretation between the new AST and the reference method divided by the number of isolates tested ${ }^{2}$.

\section{Application: food AMR survey study}


Fresh chicken breast samples ( $\mathrm{n}=35)$ were purchased from grocery stores in Vancouver, Canada. All samples were transported on ice to the lab within $2 \mathrm{~h}$ for immediate analysis. Campylobacter isolation procedure was conducted according to International Organization for Standardization protocol ISO 10272-1 with slight modifications ${ }^{12}$. First, each chicken sample was placed in a sterile sampling bag and immersed into $\mathrm{BBL}^{\mathrm{TM}}$ buffered peptone water (BD Biosciences, Canada) at a ratio of $2.5 \mathrm{~g}$ of sample per $1 \mathrm{~mL}$ of solution. After sample massaging, $1 \mathrm{~mL}$ of chicken rinse solution was transferred to a glass culture tube containing $9 \mathrm{~mL}$ of modified charcoal cefoperazone desoxycholate broth (Sigma-Aldrich, Canada). The mixture was pre-enriched at $37^{\circ} \mathrm{C}$ for $4 \mathrm{~h}$ in the microaerobic condition with shaking (175 $\mathrm{rpm}$ ), followed by incubation at $42^{\circ} \mathrm{C}$ for $48 \mathrm{~h}$ under the same shaking conditions. Then, $10 \mu \mathrm{L}$ of enriched culture was streaked onto modified charcoal cefoperazone desoxycholate agar (Sigma-Aldrich, Canada) and incubated at $42^{\circ} \mathrm{C}$ for $48 \mathrm{~h}$ in the microaerobic condition. Campylobacter-presumptive colonies with typical translucent, wet, and flat/slightly raised morphologies were collected in $10 \mu \mathrm{L}$ of sterile $\mathrm{ddH}_{2} \mathrm{O}$ for bacterial speciation. A colony multiplex PCR assay was performed for rapid identification of Campylobacter, as previously described with some modifications ${ }^{13}$. Three sets of primers were used to specifically target $16 \mathrm{~S}$ rRNA, mapA, and сеuE genes, achieving the identification of Campylobacter genus, C. jejuni, and C. coli, respectively. The primer sequences are listed in Table S3. A total of $20 \mu \mathrm{L}$ of multiplex PCR mixture was prepared that consisted of $10 \mu \mathrm{L}$ of $2 \times$ TaqFroggaMix Plus (FroggaBio, Canada), $0.5 \mu \mathrm{M}$ 16S rRNA primers, $0.5 \mu \mathrm{M}$ ceuE primers, $1 \mu \mathrm{M}$ mapA primers, $2 \mu \mathrm{L}$ of sterile $\mathrm{ddH}_{2} \mathrm{O}$, and $2 \mu \mathrm{L}$ of bacterial colony sample. DNA amplification was carried out in a C1000 Touch Thermal Cycler (Bio-Rad, USA) under the following reaction conditions: initial denaturation at $95^{\circ} \mathrm{C}$ for $10 \mathrm{~min} ; 30$ cycles at $95^{\circ} \mathrm{C}$ for $30 \mathrm{~s}$, at $55.6^{\circ} \mathrm{C}$ for $30 \mathrm{~s}$, and at $72^{\circ} \mathrm{C}$ for 30 $\mathrm{s}$; and final extension at $72^{\circ} \mathrm{C}$ for $10 \mathrm{~min}$. The PCR amplicons were checked using $1.5 \%$ agarose gel electrophoresis. Subsequently, on-chip AST was conducted for the confirmed Campylobacter isolates and the results were uploaded to the Tencent Cloud server. 


\section{TABLES}

Table S1. Comparison between IoT-microfluidic approach and the conventional AST.

\begin{tabular}{|c|c|c|c|}
\hline Characteristics & & $\begin{array}{l}\text { IoT-microfluidic } \\
\text { approach }\end{array}$ & $\begin{array}{l}\text { Conventional AST } \\
\text { (e.g., broth microdilution) }\end{array}$ \\
\hline Fixed cost & Incubator & CA $\$ 1,460$ (home-built) & $>\mathrm{CA} \$ 10,000$ (commercial) \\
\hline \multirow[t]{2}{*}{ Variable cost $^{\mathrm{a}}$} & Device & $\begin{array}{l}\text { CA } \$ 0.09 \text { (per chamber } \\
\text { in microfluidic chip) }\end{array}$ & $\begin{array}{l}\text { CA } \$ 0.02 \text { (per well in 96- } \\
\text { well plate) }\end{array}$ \\
\hline & $\begin{array}{l}\text { Growth medium \& } \\
\text { antibiotics }\end{array}$ & Cheap $(20 \mu \mathrm{L} /$ test $)$ & $\begin{array}{l}\text { Relatively pricy ( } 200 \\
\mu \mathrm{L} / \text { test })\end{array}$ \\
\hline \multirow[t]{3}{*}{$\begin{array}{l}\text { Experimental } \\
\text { operation }\end{array}$} & Sample loading & $\begin{array}{l}\text { Easy (pump injection); } \\
\text { fast ( } 3 \text { min) }\end{array}$ & $\begin{array}{l}\text { Tedious (manual pipetting); } \\
\text { Relatively slow }\end{array}$ \\
\hline & $\begin{array}{l}\text { Results } \\
\text { interpretation }\end{array}$ & $\begin{array}{l}\text { Unbiased (ML } \\
\text { algorithm) }\end{array}$ & $\begin{array}{l}\text { Potential human error } \\
\text { (visual check) }\end{array}$ \\
\hline & Automation & $\begin{array}{l}\text { Yes (except sample } \\
\text { injection) }\end{array}$ & No \\
\hline $\begin{array}{l}\text { Data integration } \\
\text { \& analysis }\end{array}$ & & $\begin{array}{l}\text { Real-time, } \\
\text { available online }\end{array}$ & Manual and delayed \\
\hline Analysis time & & $24 \mathrm{~h}$ & $24-48 \mathrm{~h}$ \\
\hline Accuracy & & Good & Good \\
\hline
\end{tabular}


Table S2. Antibiotic concentrations used in antimicrobial susceptibility testing.

\begin{tabular}{ccc}
\hline Antibiotic agent & $\begin{array}{c}\text { CLSI susceptible } \\
\text { breakpoint }(\mathrm{mg} / \mathrm{L})\end{array}$ & $\begin{array}{c}\text { CLSI resistant } \\
\text { breakpoint }(\mathrm{mg} / \mathrm{L})\end{array}$ \\
\hline Tetracycline & 4 & 16 \\
Ciprofloxacin & 1 & 4 \\
Erythromycin & 8 & 32 \\
\hline
\end{tabular}


Table S3. Primers used for Campylobacter speciation by multiplex-PCR assay

\begin{tabular}{lll}
\hline Target gene & ${\text { Oligonucleotide sequences }\left(5^{\prime}{ }^{\prime} 3^{\prime}\right)^{\text {a }}}^{\text {F }}$ & Amplicon size (bp) \\
\hline 16S rRNA & F: ATC TAA TGG CTT AAC CAT TAA AC & 857 \\
& R: GGA CGG TAA CTA GTT TAG TAT T & \\
mapA & F: CTA TTT TAT TTT TGA GTG CTT GTG & 589 \\
& R: GCT TTA TTT GCC ATT TGT TTT ATT A & \\
& & \\
ceuE & F: AAT TGA AAA TTG CTC CAA CTA TG & 462 \\
& R: TGA TTT TAT TAT TTG TAG CAG CG & \\
\hline
\end{tabular}

${ }^{\mathrm{a}} \mathrm{F}$, forward primer; $\mathrm{R}$, reverse primer. 


\section{FIGURES}
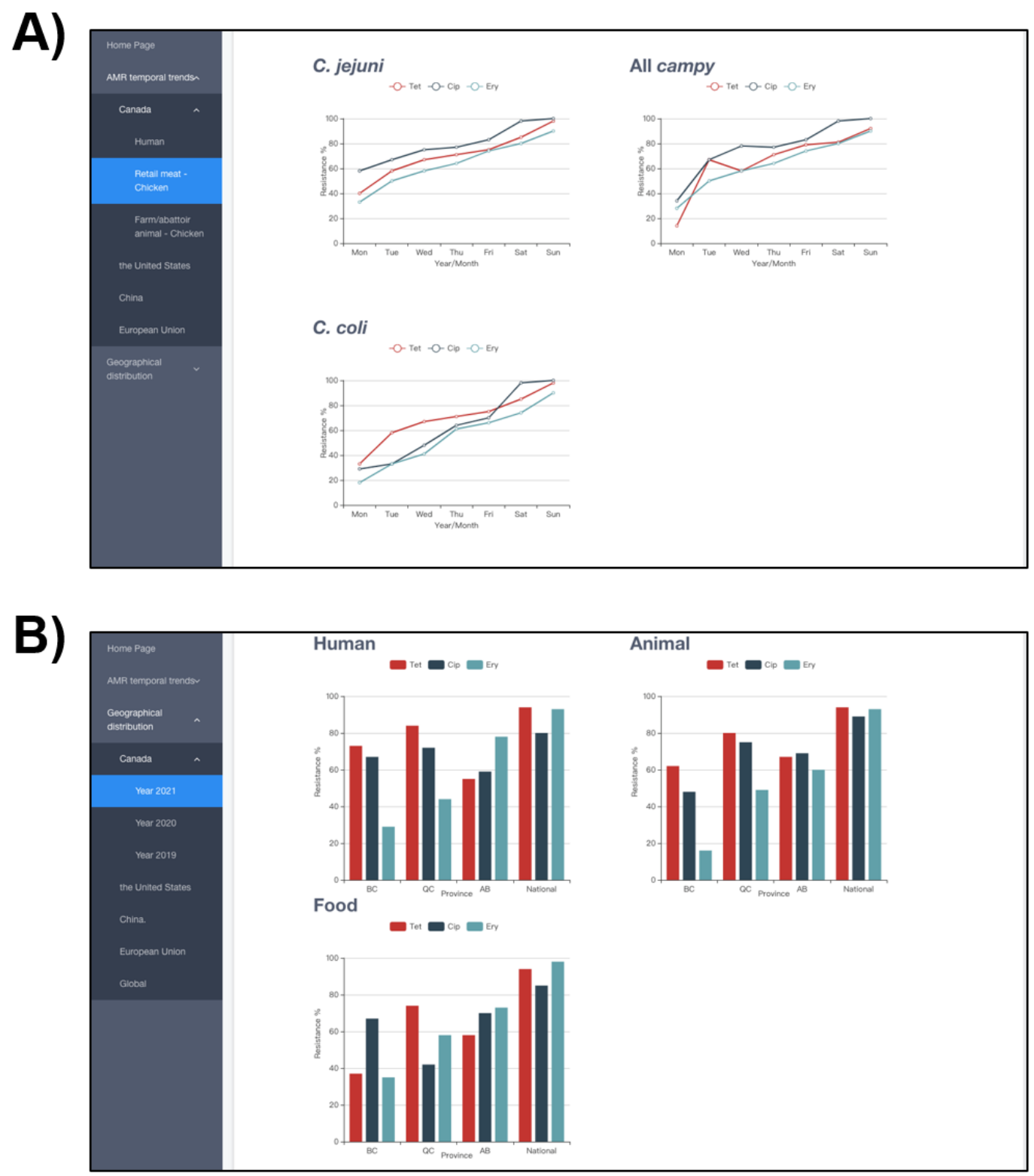

Figure S1. Web browser of the real-time AMR surveillance system. (A) Line charts are constructed to show the AMR temporal trends of Campylobacter in different isolation sources (human, animal, and foods). (B) Column charts are prepared to demonstrate the geographical distribution of AMR Campylobacter in different regions. The mock data were used to demonstrate the function of the web browser of a real-time AMR surveillance system for end-users. 


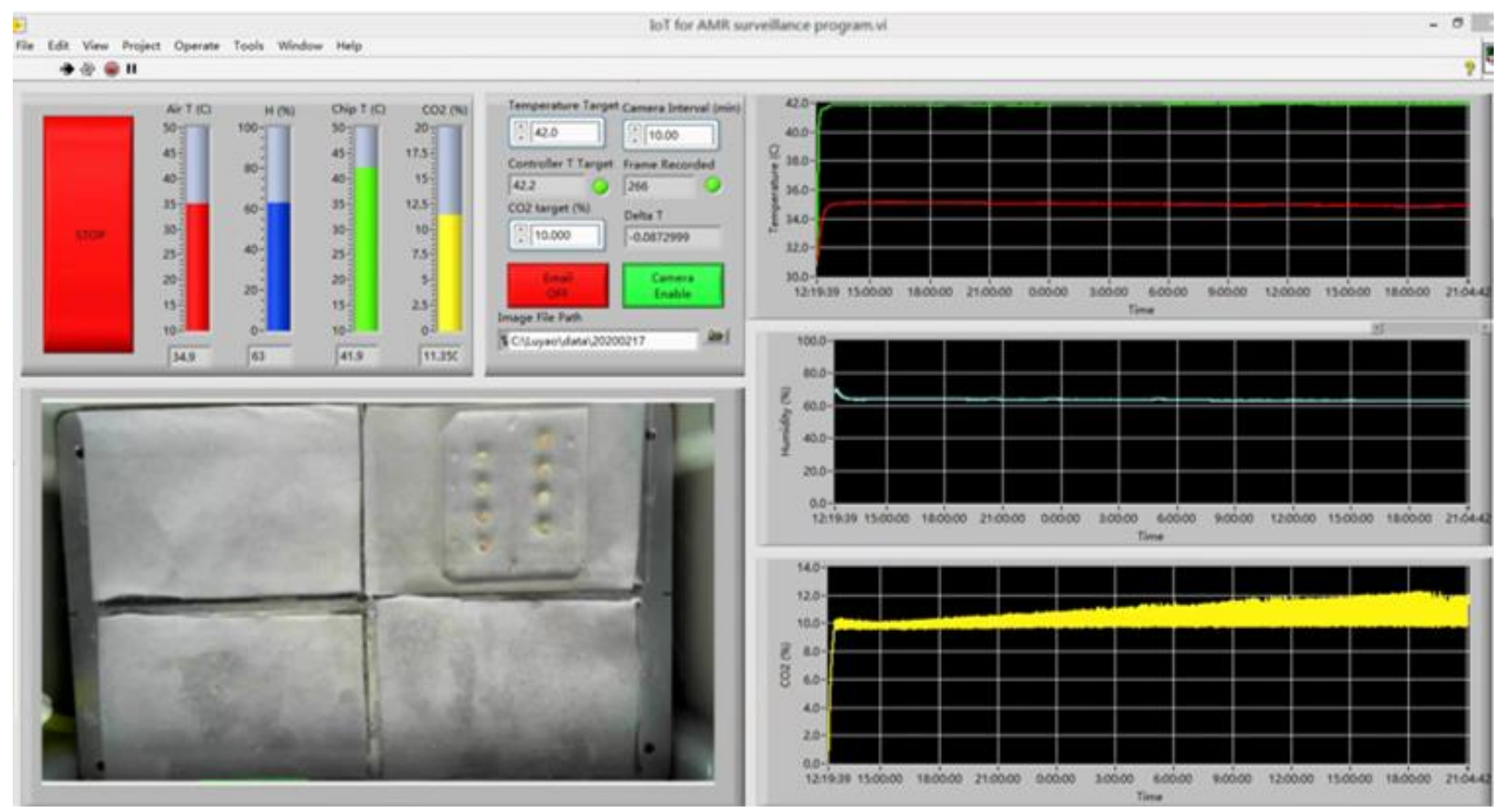

Figure S2. User interface of LabVIEW program to control IoT-based colorimetric sensing system.

Right panels from top to bottom show the real-time records of temperatures (green line, microfluidic chip temperature; red line, air temperature), relative humidity (cyan line), and $\mathrm{CO}_{2}$ concentration (yellow line). Left top panel is used to customize the cultivation parameters. Left bottom panel demonstrates the real-time images of the microfluidic chips. 


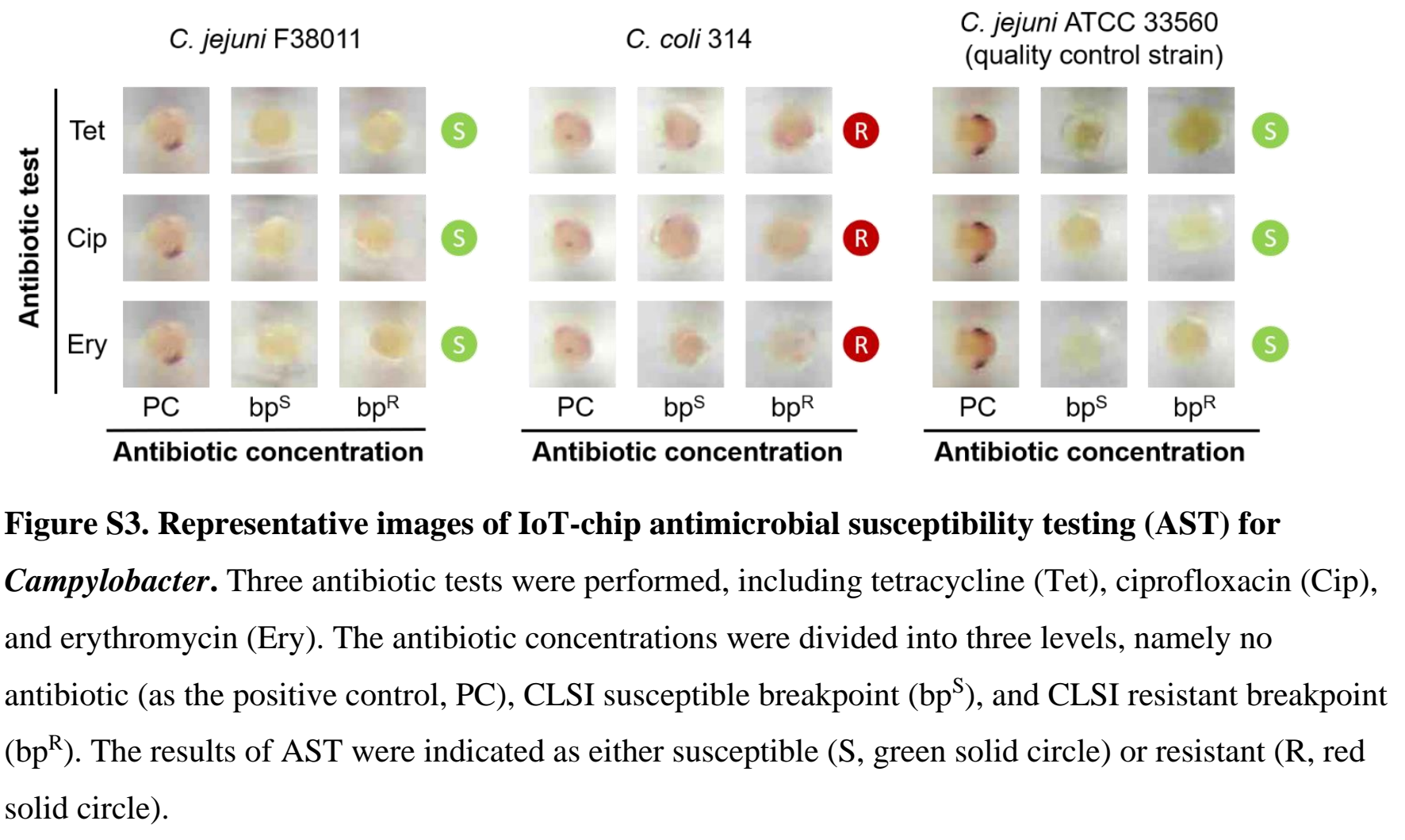


Middle layer

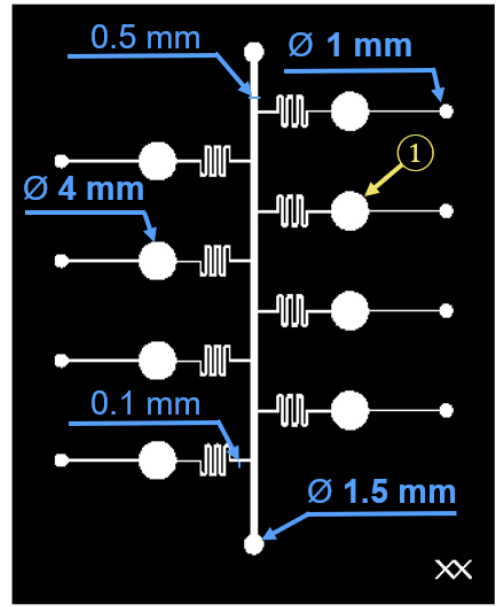

Bottom layer

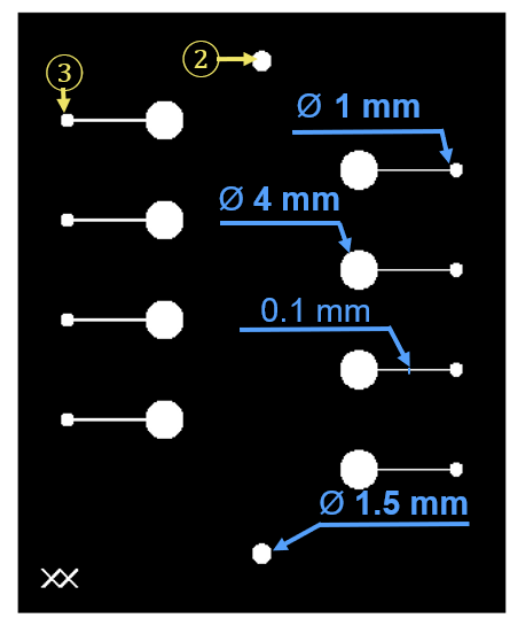

Figure S4. Mask patterns for the fabrication of middle layer (left) and bottom layer (right) of the microfluidic chips. The length and width of each layer are $48 \mathrm{~mm}$ and $40 \mathrm{~mm}$, respectively. The features marked by circled numbers indicate bacterial incubation chambers (1), inlets/outlets (2), and air vents (3). 


\section{References}

(1) Jiang, X.; Zheng, W.; Takayama, S.; Chapman, R. G.; Kane, R. S.; Whitesides, G. M. MicroScale Patterning of Cells and Their Environment. In Principles of Tissue Engineering: Fourth Edition; Academic Press, 2013; pp 359-384.

(2) Ma, L.; Petersen, M.; Lu, X. Identification and Antimicrobial Susceptibility Testing of Campylobacter Using a Microfluidic Lab-on-a-Chip Device. Appl. Environ. Microbiol. 2020, 86 (9), e00096-20.

(3) Park, S. F. The Physiology of Campylobacter Species and Its Relevance to Their Role as Foodborne Pathogens. Int. J. Food Microbiol. 2002, 74 (3), 177-188.

(4) International Telecommunication Union. Rec. ITU-R BT.601-7 Studio Encoding Parameters of Digital Television for Standard 4:3 and Wide Screen 16:9 Aspect Ratios; 2017.

(5) Mutlu, A. Y.; Kiliç, V.; Özdemir, G. K.; Bayram, A.; Horzum, N.; Solmaz, M. E. SmartphoneBased Colorimetric Detection: Via Machine Learning. Analyst 2017, 142 (13), 2434-2441.

(6) He, K.; Zhang, X.; Ren, S.; Sun, J. Deep Residual Learning for Image Recognition. In Proceedings of the IEEE Computer Society Conference on Computer Vision and Pattern Recognition; 2016; Vol. 2016, pp 770-778.

(7) Alafeef, M.; Srivastava, I.; Pan, D. Machine Learning for Precision Breast Cancer Diagnosis and Prediction of the Nanoparticle Cellular Internalization. ACS Sensors 2020, 5 (6), 1689-1698.

(8) Mercan, Ö. B.; Kılıç, V.; Şen, M. Machine Learning-Based Colorimetric Determination of Glucose in Artificial Saliva with Different Reagents Using a Smartphone Coupled MPAD. Sensors Actuators, B Chem. 2021, 329, 129037-129038.

(9) Narvaez-Bravo, C.; Taboada, E. N.; Mutschall, S. K.; Aslam, M. Epidemiology of Antimicrobial Resistant Campylobacter spp. Isolated from Retail Meats in Canada. Int. J. Food Microbiol. 2017, 253, 43-47.

(10) Han, X.; Zhu, D.; Lai, H.; Zeng, H.; Zhou, K.; Zou, L.; Wu, C.; Han, G.; Liu, S. Prevalence, Antimicrobial Resistance Profiling and Genetic Diversity of Campylobacter jejuni and Campylobacter coli Isolated from Broilers at Slaughter in China. Food Control 2016, 69, 160170.

(11) Clinical and Laboratory Standards Institute (CLSI). Methods for Antimicrobial Dilution and Disk Susceptibility Testing of Infrequently Isolated or Fastidious Bacteria, 3rd Edition. CLSI M45. Wayne, PA, USA 2016. 
(12) Ma, H.; Su, Y.; Ma, L.; Ma, L.; Li, P.; Du, X.; Golz, G.; Wang, S.; Lu, X. Prevalence and Characterization of Campylobacter jejuni Isolated from Retail Chicken in Tianjin, China. J. Food Prot. 2017, 80 (6), 1032-1040.

(13) Denis, M.; Soumet, C.; Rivoal, K.; Ermel, G.; Blivet, D.; Salvat, G.; Colin, P. Development of a m-PCR Assay for Simultaneous Identification of Campylobacter jejuni and C. coli. Lett. Appl. Microbiol. 1999, 29 (6), 406-410. 